\title{
Increase in Risk and its Effects on Welfare and Optimal Policies in a Dynamic Setting: The Case of Global Pollution
}

\author{
Quentin Grafton $^{\mathrm{a}}$, Tom Kompas ${ }^{\mathrm{a}}$ and Ngo Van Long ${ }^{\mathrm{b}}$ \\ ${ }^{a}$ Crawford School, Australian National University, Canberra, A.C.T. 0200, Australia. \\ ${ }^{\mathrm{b}}$ Department of Economics, McGill University, 855 Sherbrooke St West, Montreal, QC, Canada H3A2T7.
}

This paper studies the effects of an increase in risk on welfare and optimal policies in a stochastic dynamic model of global pollution. In a first step, we focus on the case of a single decision maker, and make use of an approach pioneered by Kimball (2014) for studying the impact of a marginal change in risk in optimal stochastic control models. Using a simple model with only one state variable and one control variable, we show how the optimal carbon tax responds to an increase in risk. It is found that the third derivative of the decay function of the stock of pollution may play a decisive role. In a second step, we investigate the extent to which Kimball's approach may be extended to the case of stochastic dynamic games. We show how strategic interactions complicate the task of evaluating the effects of an increase in risk. Interestingly, in a dynamic model of the tragedy of the commons, we find that an increase in risk can increase welfare even though all agents are risk averse. The reason is that higher risk can cause agents to be more conservative, and this mitigates the tragedy of the commons.

The Geneva Risk and Insurance Review (2014) 39, 40-64. doi:10.1057/grir.2014.5

Keywords: comparative dynamics; risk; pollution; tragedy of the commons

Article submitted 3 December 2013; accepted 28 April 2014

\section{Introduction}

The effects on an increase in risk on the behaviour of economic agents are well understood in static settings. The same cannot be said when agents maximise repeatedly over time, and in particular when they interact strategically with each other in a dynamic setting. There are specific dynamic models in which the effects of risks are assessed, but they mostly rely on specific functional forms. ${ }^{1}$ A notable exception is the paper by

\footnotetext{
${ }^{1}$ For example, Merton (1990); Turnovsky (2000, Chs. 15 and 16); van den Bremer et al. (2013).
} 
Kimball, ${ }^{2}$ in which the author offers a general framework for exploring the effects of a marginal increase in risk on the behaviour of a single decision-maker when the dynamics of the capital stock are subject to random disturbance.

The purpose of the present paper is two-fold. First, we study the effect of an increase in risk on welfare and on the optimal policy in the case of global pollution, assuming that policies are in the hand of a single decision-maker. This assumption allows us to make use of Kimball's methods. Second, we investigate how a marginal increase in risk may affect welfare when the global common is exploited by several non-cooperative players interacting strategically in a dynamic setting. We find that, to some extent, Kimball's methods can be generalised to the dynamic game setting. Under additional assumptions, we are able to show that an increase in risk may increase the welfare of the players.

Kimball's method makes use of a dynamic version of the envelope theorem, a tool that has been explored by Caputo, ${ }^{3}$ LaFrance and Barney, ${ }^{4}$ Ling and Caputo, ${ }^{5}$ Caputo and Ling, ${ }^{6}$ and others, who focus on deterministic problems. Our pollution control problems illustrate the usefulness, as well as the limitations, of the dynamic envelope theorem in the analysis of the effects of an increase in risk in stochastic control problems and stochastic differential games.

The next section deals with the case of a single decision-maker. Using a simple model of climate change, we show how the optimal carbon tax responds to an increase in risk. It is found that the sign of third derivative of the decay function of the pollution stock may play a decisive role, in line with the well-known result on precautionary saving where the sign of the third derivative of the utility function is crucial. ${ }^{7}$ The subsequent section extends the model to the case of dynamic games involving several intertemporal optimisers acting strategically. We show how strategic interactions complicate the task of evaluating the effects of an increase in risk. Interestingly, in a dynamic model of the tragedy of the commons, we find that a marginal increase in risk can increase welfare even though all agents are risk averse. The reason is that higher risk can cause agents to be more conservative, and this mitigates the tragedy of the commons.

\section{The case of a single decision-maker}

For the moment, we abstract from strategic interactions. We present below a simple formulation of intertemporal optimisation under risk. Our formulation is intentionally

\footnotetext{
${ }^{2}$ Kimball (2014).

${ }^{3}$ Caputo (1990, 2003, 2005, 2007).

${ }^{4}$ LaFrance and Barney (1991).

${ }^{5}$ Ling and Caputo (2011).

${ }^{6}$ Caputo and Ling (2013).

${ }^{7}$ Kimball (1990).
} 
kept very simple: it involves one state variable, and there is only one control variable available to the single decision-maker. ${ }^{8}$ Using this formulation, we develop a toolbox that helps determine the effects of an increase in risk when the system is initially in a neighbourhood of a risk-free steady state. Using Kimball's approach, we show how one may derive simple formulas to evaluate the effects of a marginal change in risk on the "welfare" (i.e. on the optimal value of the programme) and on the "policy function" when there is only one control variable. This analysis will be followed by a specific application to the economics of climate change. In particular, we show the importance of the sign of the third derivative of the decay function of the pollution stock.

\section{Effect of an increase in risk: A toolbox for problems with one control variable}

We restrict attention to a dynamic environment involving only one state variable, one control variable and one source of risk. ${ }^{9}$ Time is continuous and is denoted by $t$. Let $s(t)$ and $c(t)$ denote the value of the state variable and the control variable at time $t$. Suppose that in the absence of risk, the rate of change of the state variable depends only on the current levels of the state variable and the control variable:

$$
\frac{\mathrm{d} s(t)}{\mathrm{d} t}=G(s(t), c(t))
$$

where $G(s, c)$ is a known function with continuous third derivatives. This equation is interpreted as follows. Consider a very small interval of time of length $\mathrm{d} t>0$. Between time $t$ and time $t+\mathrm{d} t$, the control variable is approximately constant, and is equal to $c(t)$. Then, in the absence of risk, the change in the state variable over this time interval is approximately

$$
\mathrm{d} s(t)=G(s(t), c(t)) \mathrm{d} t
$$

Now we introduce risk. For simplicity, suppose the risk is in the form of an additional source of change in $s$. This source is driven by a stochastic process $z(t)$, with stochastic increment $\mathrm{d} z$ over the small time interval $\mathrm{d} t$. We adopt the standard assumption that $\mathrm{d} z$ is normally distributed, with zero mean, and variance $\mathrm{d} t$. We assume that the stochastic component of the change in $s$ over the small interval $\mathrm{d} t$ is equal to $\sqrt{\mu} \sigma(s(t)) \mathrm{d} z$, where $\sigma(s)>0$ is a known function of $s$, and $\mu$ is scale parameter. Then the transition equation takes the form of a simple stochastic

\footnotetext{
${ }^{8}$ Our formulation is a special case of Kimball's: He allows the decision-maker to have several control variables, which result in more complex expressions.

${ }^{9}$ The exposition in this subsection summarises some key results for easy reference; for more general results, see Kimball (2014).
} 
differential equation ${ }^{10}$ :

$$
\mathrm{d} s(t)=G(s(t), c(t)) \mathrm{d} t+\sqrt{\mu} \sigma(s(t)) \mathrm{d} z .
$$

The variance of the non-deterministic component of the change in $s$ is $\mu \sigma^{2} \mathrm{~d} t$.

With this formulation, the riskless situation corresponds to the case where $\mu=0$. An increase in $\mu$ represents an increase in risk.

Suppose the decision maker has an instantaneous utility function $U(s, c)$, and his utility discount rate is $r>0$. The objective of the decision maker is to maximise the expected value of the discounted stream of utility:

$$
\max _{c} E \int_{0}^{\infty} e^{-r t} U(s(t), c(t)) \mathrm{d} t
$$

subject to the transition equation (1), and the initial condition $s(0)=s_{0}$.

As explained in standard references, ${ }^{11}$ to find the optimal solution, the standard approach is to determine a value function $V(s, \mu)$ and a control rule $c(s, \mu)$ such that the following Hamilton-Jacobi-Bellman (HJB) equation is satisfied:

$$
r V(s, \mu)=\max _{c}\left[U(s, c)+V_{s} G(s, c)+\frac{1}{2} \mu \sigma^{2}(s) V_{s s}\right] .
$$

In addition, for sufficiency, the following transversality condition is imposed (p. 229) $)^{11}$

$$
\lim \sup _{t \rightarrow \infty} e^{-r t} E_{c} V(s(t), \mu) \leq 0 .
$$

In the literature, the right-hand side of the HJB equation (2) is not given a name. Since it resembles the current-value Hamiltonian function of the standard optimal control problem, let us call it the "pseudo-Hamiltonian" and denote it by $K(s, c, \mu){ }^{12}$ We record this definition below:

$$
K(s, c, \mu) \equiv U(s, c)+V_{s} G(s, c)+\frac{1}{2} \mu \sigma^{2}(s) V_{s s}
$$

\footnotetext{
${ }^{10}$ For a brief introduction to stochastic differential equations, see Dockner et al. (2000, Ch. 8), and Merton (1990, Ch. 2).

${ }^{11}$ For example, Dockner et al. (2000).

12 The Hamiltonian function is slightly different from the pseudo-Hamiltonian: In the Hamiltonian function, $V_{s}$ is replaced by a shadow price $\psi(t)$ which is a function of time, not a function of $s$. Of course, once the optimal path is found, $\psi(t)$ is equal to $V_{s}(s(t))$ along the optimal path. See, for example, Leonard and Long (1992).
} 
The optimal control $c$ must maximise the pseudo-Hamiltonian; this gives the firstorder condition $^{13}$

$$
K_{c}(s, c(s, \mu), \mu)=0 .
$$

Applying the implicit function theorem to this equation, we obtain the optimal response of the control variable to an increase in $s$ :

$$
\frac{\partial c}{\partial s}=-K_{s c}\left[K_{c c}\right]^{-1}=-\left[U_{s c}+V_{s} G_{s c}+V_{s s} G_{c}\right]\left[U_{c c}+V_{s} G_{c s}\right]^{-1} .
$$

The response of the control variable to an increase in risk is:

$$
\frac{\partial c}{\partial \mu}=-K_{\mu c}\left[K_{c c}\right]^{-1}=-V_{\mu s} G_{c}\left[U_{c c}+V_{s} G_{c s}\right]^{-1} .
$$

From these expressions, it is clear that in order to determine the effect of an increase in risk on the optimal control in some neighbourhood of the risk-free steady state, we must calculate the derivatives $V_{s}, V_{s s}$, and $V_{\mu s}$ in that neighbourhood.

Let us denote by $s^{*}$ the steady state in the risk-free scenario, that is, when $\mu=0$.

With $\mu=0$, the HJB equation is simply

$$
r V(s)=\max _{c}\left[U(s, c)+V_{s} G(s, c)\right]
$$

and maximising the pseudo-Hamiltonian with respect to $c$ yields

$$
U_{c}+V_{s} G_{c}=0 \Rightarrow V_{s}-\frac{U_{c}}{G_{c}} .
$$

Then

$$
r V(s)=U(s, c(s))+V_{s} G(s, c(s)) .
$$

Differentiating the above equation with respect to $s$, and applying the envelope theorem, we obtain

$$
r V_{s}=U_{s}+V_{s} G_{s}+G V_{s s} .
$$

At the steady state, $G\left(s^{*}, c\left(s^{*}\right)\right)=0$, and we obtain

$$
r V_{s}\left(s^{*}\right)=U_{s}\left(s^{*}, c\left(s^{*}\right)\right)+V_{s}\left(s^{*}\right) G_{s}\left(s^{*}, c\left(s^{*}\right)\right) .
$$

Thus

$$
V_{s}\left(s^{*}\right)=\frac{U_{s}\left(s^{*}, c\left(s^{*}\right)\right)}{r-G_{s}\left(s^{*}, c\left(s^{*}\right)\right)} .
$$

\footnotetext{
${ }^{13}$ We assume that $K$ is quasi-concave in $c$, so that the second-order condition is satisfied, that is, $K_{c c}<0$ whenever $K_{c}=0$.
} 
Combining this equation with the FOC (5), we obtain the equation that characterises the risk-free steady state:

$$
-\frac{U_{c}\left(s^{*}, c\left(s^{*}\right)\right)}{G_{c}\left(s^{*}, c\left(s^{*}\right)\right)}=\frac{U_{s}\left(s^{*}, c\left(s^{*}\right)\right)}{r-G_{s}\left(s^{*}, c\left(s^{*}\right)\right)} .
$$

We assume that Eq. (6) has a unique solution. As usual, we take it that $s^{*}$ has the saddle-point property. Along the stable-branch of saddle point, the optimal control can be expressed as function of $s$, that is, $c=c(s)$. Then the optimal rate of change in $s$ is $G(s, c(s))$. The "reduced-form policy function" is then $\dot{s}(s)=G(s, c(s))$. Stability in the saddle-point sense implies that, in a neighbourhood of the steady state $s^{*}$, the slope of the reduced-form policy function is negative. We denote this slope by $-\gamma$ :

$$
-\gamma \equiv G_{s}\left(s^{*}, c\left(s^{*}\right)\right)+G_{c}\left(s^{*}, c\left(s^{*}\right)\right) c^{\prime}\left(s^{*}\right)<0 .
$$

Now we introduce risk by considering an increase in $\mu$ from zero to some $\mu>0$. We ask the following question: suppose we are initially at (or near) the risk-free steady state $s^{*}$. What is the effect of an increase in risk on welfare? The answer is provided by the following result ${ }^{14}$ :

Result 1: Starting at or near the risk-free steady state $s^{*}$, a marginal increase in risk will reduce welfare if and only if the value function $V(s, \mu)$ is locally strictly concave in s. The magnitude of this welfare-reducing effect varies in direct proportion to $\sigma^{2}\left(s^{*}\right)$, and varies inversely with the discount rate $r$. More precisely,

$$
V_{\mu}\left(s^{*}, 0\right)=\frac{1}{2 r} \sigma^{2}\left(s^{*}\right) V_{s s}\left(s^{*}, 0\right) .
$$

This result is intuitively appealing. A concave $V$ suggests risk aversion. Then an increase in risk reduces welfare. However, it is important to note that the function $V$ is a not a primitive, and therefore we cannot really assume that $V$ is concave. The primitives of the model are the functions $U$ and $G$. Without knowing the local concavity or convexity of $U$ and $G$, we cannot say anything about the local concavity or convexity of $V$. Fortunately, the following sufficiency results are available.

Fact 1: If the state variable is a "bad", that is, an increase in the initial endowment of that variable will reduce welfare, then a set of sufficient conditions for $V_{s s}$ to be negative is that (i) $U(s, c)$ is strictly concave in $(s, c)$ and (ii) $G(s, c)$ is strictly convex in $(s, c)$.

Fact 2: If the state variable is a "good", that is, an increase in the initial endowment of that variable will increase welfare, then a sufficient condition for $V_{s s}$ to be negative is that both $U(s, c)$ and $G(s, c)$ are strictly concave in $(s, c){ }^{15}$

\footnotetext{
${ }^{14}$ We will not prove Results 1-3, and Facts 3-4, because they can be inferred from the more general results in Kimball (2014).

15 For a proof of Facts 1 and 2, see, for example, Long (1979).
} 
Facts 1 and 2 are concerned with global rather than local properties, and tell us about the sign of $V_{s s}$ but nothing about its magnitude. In order to obtain its magnitude, we resort to the following result:

Fact 3: Suppose that the following inequality holds at the risk-free steady state ${ }^{16}$ :

$$
U_{s s}\left(s^{*}, c^{*}\right)+V_{s}\left(s^{*}, 0\right) G_{s s}\left(s^{*}, c^{*}\right)<\left[K_{s c}\left(s^{*}, c^{*}, 0\right)\right]^{2}\left[K_{c c}\left(s^{*}, c^{*}, 0\right)\right]^{-1} .
$$

Then $V_{s s}\left(s^{*}, 0\right)$ is equal to the negative root of the following quadratic equation in $\rho$

$$
A \rho^{2}+B \rho+C=0
$$

where

$$
\begin{gathered}
A \equiv-\left(G_{c}\right)^{2}\left[K_{c c}\left(s^{*}, c^{*}, 0\right)\right]^{-1}>0, \\
B \equiv 2 G_{s}-r-\left(2 G_{x}\right)\left[K_{s c}\left(s^{*}, c^{*}, 0\right)\right]\left[K_{c c}\left(s^{*}, c^{*}, 0\right)\right]^{-1}, \\
C \equiv U_{s s}+V_{s} G_{s s}-\left[K_{s c}\left(s^{*}, c^{*}, 0\right)\right]^{2}\left[K_{c c}\left(s^{*}, c^{*}, 0\right)\right]^{-1}<0,
\end{gathered}
$$

where all derivatives are evaluated at $(s, c, \mu)=\left(s^{*}, c^{*}, 0\right)$.

Let us turn to the effect of an increase in risk on the marginal value of the stock. In other words, we want to determine the sign of the cross derivative $V_{\mu s}$. We have the following result:

Result 2: Starting at the steady state $s^{*}$ of the risk-free scenario, the effect of an increase in risk on the marginal value of the stock is given by the formula

$$
V_{\mu s}=\frac{V_{s s} \sigma\left(s^{*}\right) \sigma^{\prime}\left(s^{*}\right)+V_{s s s} \frac{\sigma^{2}\left(s^{*}\right)}{2}}{r+\gamma} .
$$

Thus, an increase in risk will decrease the marginal value of the stock at $s^{*}$ if $V_{s s}<0, \sigma^{\prime}\left(s^{*}\right)>0$, and $V_{s s s} \leqslant 0$. But if $V_{s s s}>0$, then it is possible that an increase in risk may increase the marginal value of the stock at $s^{*}$.

It should be carefully noted that Result 2 says that a direct way to know the sign of $V_{\mu s}$ is to determine the third-order derivative $V_{s s s}$ as well $V_{s s}$ and $\sigma^{\prime}\left(s^{*}\right)$. How do we calculate $V_{\text {sss }}$ ? The following fact is useful.

Fact 4: The derivative $V_{\text {sss }}$ can be computed as follows:

$$
(r+3 \gamma) V_{s s s}=M+P V_{s}+Q V_{s s}
$$

\footnotetext{
${ }^{16}$ This condition holds if the maximised Hamiltonian function is locally strictly concave in the state variable.
} 
where

$$
\begin{aligned}
M & \equiv U_{s s s}+3 q U_{s s c}+3 q^{2} U_{s c c}+q^{3} U_{c c c} \\
P & \equiv G_{s s s}+3 q G_{s s c}+3 q^{2} G_{s c c}+q^{3} G_{c c c} \\
Q & \equiv 3\left(G_{s s}+2 q G_{s c}+q^{2} G_{c c}\right) \\
q & \equiv \frac{\partial c(s, 0)}{\partial s}=-\left(U_{s c}+V_{s} G_{s c}+V_{s s} G_{c}\right)\left(U_{c c}+V_{s} G_{c c}\right)^{-1}
\end{aligned}
$$

Finally, the effect of an increase in risk on the control variable at the risk-free steady state can be calculated as follows.

Result 3: If an increase in risk increases the marginal value of the stock (i.e. if $V_{\mu s}>0$ ), then it increases the control variable (provided that the control variable has a positive contribution to the growth rate of the stock, i.e. provided that $G_{c}>0$ ):

$$
\begin{aligned}
\frac{\partial c}{\partial \mu} & =-K_{\mu c}\left[K_{c c}\right]^{-1}=-V_{\mu s} G_{c}\left[U_{c c}+V_{s} G_{c c}\right]^{-1} \\
& =\left(-G_{c}\right) \frac{V_{s s} \sigma\left(s^{*}\right) \sigma^{\prime}\left(s^{*}\right)+V_{s s s} \frac{\sigma^{2}\left(s^{*}\right)}{2}}{r+\gamma}\left[U_{c c}+V_{s} G_{c c}\right]^{-1} .
\end{aligned}
$$

\section{The response of optimal emissions tax to an increase in risk}

We consider the case of optimal control of the time path of a "public bad": the global pollution stock, $s(t)$. We use the methods expounded in the preceding subsection to investigate the effect of a marginal increase in risk on social welfare, and on the optimal policy (interpreted as the optimal carbon tax). We show that the effect on optimal policy depends on the third derivatives of three functions: the damage function, the utility function, and the decay function of the pollution stock, as well as on the first derivative of the variance of risk with respect to the stock of pollution.

Suppose the world consists of a continuum of identical households that are both producers and consumers. Each household is a point on the continuum $[0, L]$. We interpret $L$ to be the population size. (In what follows, we normalise by setting $L=1$ for simplicity.)

For each unit of output produced, one unit of pollution is emitted as a by-product. Each household consumes its own output, $c(t)$ at time $t$. This yields a flow $u(c(t))$ of utility for each household. The utility function $u(c)$ is strictly concave, with $u^{\prime}(c)>0$ and $u^{\prime \prime}(c)<0$. The stock of pollution inflicts a damage cost $D(s(t))$ at time $t$ to each household. The damage function $D(s)$ is increasing and strictly convex, with 
$D(0)=0, D^{\prime}(s) \geqslant 0$, and $D^{\prime \prime}(s)>0$ for $s>0$. The transition equation is assumed to take the form

$$
\mathrm{d} s(t)=[c(t)-\delta(s(t))] \mathrm{d} t+\sqrt{\mu} \sigma(s(t)) \mathrm{d} z .
$$

The right-hand side is the sum of a deterministic component, $[c(t)-\delta(s(t))] \mathrm{d} t$, and a stochastic component, $\sqrt{\mu} \sigma(s(t)) \mathrm{d} z$. We assume that $\sigma^{\prime}(s) \geqslant 0, \sigma^{\prime}(0)>0$, and $\sigma(0)=0$.

The function $\delta(s)$ stands for the net natural decay of the pollution stock. We assume that $\delta(s)$ is concave (possibly linear), with $\delta(0)=0$ and $\delta^{\prime}(s)>0$ for small values of $s .{ }^{17}$ In the theoretical literature, most authors use the simplifying function that $\delta(s)$ is linear, that is, $\delta(s)=\varepsilon s$, where $\varepsilon>0$ is called the constant proportional rate of decay. In the context of climate change, it is possible that for large values of $s$, the function $\delta(s)$ is negative; this could represent feedback effects, such as the additional emissions from the melting of the permafrost when the global average temperature has passed a certain threshold. See, for example, Sandal et al. ${ }^{18}$

We consider two cases relating to the natural decay function $\delta(s)$.

Case 1: (weak feedback effect) In this case, $\delta(s)$ is an increasing function. The proportional rate of decay, $\delta(s) / s$, is always positive, though it may be decreasing in $s$.

Case 2: (strong feedback effect) In this case, $\delta(s)$ becomes negative after the stock passes a certain threshold $\bar{s}>0$. Then the concavity of $\delta(s)$ implies that there exists a value $\hat{s} \in(0, \bar{s})$ such that $\delta^{\prime}(\hat{s})=0$.

For an example of Case 1, we may suppose the following functional form

$$
\delta(s)=\frac{s}{1+s}, \delta^{\prime}>0, \delta^{\prime \prime}<0, \delta^{\prime \prime \prime}>0 .
$$

For an example of Case 2, we may consider the functional form

$$
\delta(s)=\varepsilon s-\frac{s^{2}}{2}+\xi\left[1-e^{-\omega s}\right], \varepsilon>0, \xi>0, \omega>0 .
$$

Note that this implies $\delta^{\prime}(0)>0, \delta^{\prime \prime}(s)<0$ and $\delta^{\prime \prime \prime}(s)>0$.

The social planner wants to maximise the expected value of the flow of social welfare:

$$
\max _{c \geq 0} E \int_{0}^{\infty} e^{-r t}[u(c(t))-D(s(t))] \mathrm{d} t
$$

subject to the transition equation and the initial condition $s(0)=s_{0}$. We assume that $s_{0}$ is sufficiently small, so that $\delta\left(s_{0}\right)>0$.

\footnotetext{
${ }^{17}$ The concavity of $\delta(s)$ and $u(c)$, together with the convexity of $D(s)$, ensures that the sufficient conditions for an optimal are satisfied.

${ }^{18}$ Sandal et al. (2003).
} 
The pseudo-Hamiltonian is

$$
K(s, c, \mu) \equiv u(c)-D(s)+V_{s}[c(t)-\delta(s(t))]+\frac{1}{2} \mu \sigma^{2}(s) V_{s s} .
$$

Maximising $K$ with respect to $c$ yields the first-order condition

$$
u^{\prime}(c)+V_{s}(s, \mu)=0 .
$$

This equation gives $c$ as a function of $(s, \mu)$ :

$$
u^{\prime}(c(s, \mu))=-V_{s}(s, \mu) \Rightarrow c(s, \mu)=u^{\prime-1}\left[-V_{s}(s, \mu)\right] .
$$

This indicates that $-V_{s}(s, \mu)$ is the required carbon tax to induce households to choose the correct emission level $c(s, \mu)$.

Applying the formula (6) for identifying the steady state, we obtain

$$
u^{\prime}\left(\delta\left(s^{*}\right)\right)=\frac{D^{\prime}\left(s^{*}\right)}{r+\delta^{\prime}\left(s^{*}\right)} .
$$

The left-hand side is the marginal utility of the steady-state consumption level. The right-hand side is the present value of the stream of damages that a marginal increase in emissions would inflict to society. It is equal to the optimal tax on emissions at the steady state. Notice that in the denominator, the marginal decay rate, $\delta^{\prime}\left(s^{*}\right)>0$, is added to the discount rate, $r$. This is because only a fraction of unit of today's $\mathrm{CO}_{2}$ emissions will remain in the atmosphere after one year, and even a smaller fraction of it will remain after two years, and so on. The higher the decay rate, the lower the present value of the stream of damages caused by a marginal unit of today's emissions.

In order to ensure that the steady state is unique, we make the following assumptions:

Assumption Al: In the strong feedback case, where $\delta^{\prime}(s)<0$ for all $s>\hat{s}$, we assume

$$
A(c) r<\frac{\delta^{\prime \prime}(s)}{\delta^{\prime}(s)} \text { for all } s>\hat{s},
$$

where $c$ is evaluated at $c=\delta(s)$ and $A(c)$ is the absolute risk-aversion $\left(A(c)=-u^{\prime \prime} / u^{\prime}\right){ }^{19}$ Assumption A2: In the strong feedback case, we assume that the marginal damage cost at the pollution stock level $\bar{s}$ is sufficient large, such that

$$
D^{\prime}(\bar{s})>\left[r+\delta^{\prime}(0)\right] u^{\prime}(\delta(0))
$$

\footnotetext{
${ }^{19}$ Clearly, if $A(c)$ is bounded and $r$ is sufficiently small, then condition (12) is satisfied.
} 
50

Lemma 1: The risk-free steady-state $s^{*}$ is unique in the weak feedback case. In the strong feedback case, $s^{*}$ is unique under Assumptions A1 and A2.

Proof To prove the uniqueness of $s^{*}$, re-write Eq. (11) as follows

$$
D^{\prime}\left(s^{*}\right)=\left[r+\delta^{\prime}\left(s^{*}\right)\right] u^{\prime}\left(\delta\left(s^{*}\right)\right) .
$$

The left-hand side (LHS) is monotone increasing in $s^{*}$. Since $D^{\prime}(0)=0$, it holds that, at $s=0$, the intercept of the graph of the LHS is below the intercept of the graph of the RSH. In the weak feedback case, the RHS is clearly decreasing in $s$. Hence the two curves intersect each other exactly once. Consider now the strong feedback case. Note that the right-hand side is monotone decreasing in $s^{*}$ iff

$$
\delta^{\prime \prime}(s) u^{\prime}(\delta(s))+\left[r+\delta^{\prime}(s)\right] u^{\prime \prime}(\delta(s)) \delta^{\prime}(s)<0 .
$$

Inequality (13) obviously holds for all $s \leq \hat{s}$. For $s \in(\hat{s}, \bar{s})$, inequality (13) holds iff

$$
-\delta^{\prime \prime}(s) u^{\prime}>\left(-u^{\prime \prime}\right)\left[r+\delta^{\prime}(s)\right]\left[-\delta^{\prime}(s)\right] \text { for all } s \in(\hat{s}, \bar{s}) .
$$

Now, since $\delta^{\prime}(s)<0$ for $s \in(\hat{s}, \bar{s})$, we have

$$
\left[r+\delta^{\prime}(s)\right]\left[-\delta^{\prime}(s)\right]<r\left[-\delta^{\prime}(s)\right] \text { for all } s \in(\hat{s}, \bar{s}) .
$$

It follows that, when Assumption A1 holds, so that $-\delta^{\prime \prime}(s) u^{\prime}>\left(-u^{\prime \prime}\right) r\left[-\delta^{\prime}(s)\right]$ for all $s \in(\widehat{s}, \bar{s})$, then condition (13) is satisfied also for $s \in(\hat{s}, \bar{s})$. Assumption A2 then ensures that the intersection point $s^{*}$ is to the left of $\bar{s}$.

We are now ready to find out (i) whether, starting from the riskless steady state, an increase in risk will reduce welfare, and (ii) whether an increase in risk will lead to an increase or decrease in the optimal carbon tax in some neighbourhood of the stock level $s^{*}$.

To answer question (i), we note that, from Eq. (7), an increase in risk will reduce welfare if and only if $V_{s s}\left(s^{*}, 0\right)<0$. Since the optimal control problem at hand satisfies the conditions stated in Fact 1 , we know that $V_{s s}$ is negative. To calculate the magnitude of $V_{s s}\left(s^{*}, 0\right)$, we make use of formula (8). We obtain

$$
V_{s s}\left(s^{*}, 0\right)=\frac{r+2 \delta^{\prime}\left(s^{*}\right)-\sqrt{\left(r+2 \delta^{\prime}\left(s^{*}\right)\right)^{2}-4 u^{\prime \prime}\left(c^{*}\right)\left(D^{\prime \prime}\left(s^{*}\right)-\delta^{\prime \prime}\left(s^{*}\right) \frac{D^{\prime}\left(s^{*}\right)}{r+\delta^{\prime}\left(s^{*}\right)}\right)}}{-2 u^{\prime \prime}\left(c^{*}\right)}<0 .
$$

Proposition 1 (Effect of an increase in risk on welfare) Assume that (i) $u(c)$ is strictly concave, (ii) $D(s)$ is strictly convex, and (iii) $\delta(s)$ is linear or concave. Then, starting from any point in a small neighbourhood of the risk-free steady state, an increase in risk will reduce welfare. 
Remark: From Eq. (14), we see that if $D^{\prime \prime}=0$ and $\delta^{\prime \prime}=0$, then an increase in risk will have no effect on welfare.

To answer question (ii), we note that the carbon tax is equal to $-V_{s}(s, \mu)$. An increase in risk will lead to an increase in the carbon tax in a neighbourhood of $s^{*}$ if and only if $-V_{s \mu}\left(s^{*}, 0\right)$ is positive (i.e. iff an increase in $\mu$ leads to an increase $-V_{s}$ ).

Let us compute $-V_{s \mu}\left(s^{*}, 0\right)$. From Eq. (9),

$$
-V_{\mu s}\left(s^{*}, 0\right)=\frac{-V_{s s}\left(s^{*}, 0\right) \sigma\left(s^{*}\right) \sigma^{\prime}\left(s^{*}\right)-V_{s s s}\left(s^{*}, 0\right) \frac{\sigma^{2}\left(s^{*}\right)}{2}}{r+\gamma} .
$$

If $V_{s s s}<0$, then an increase in risk will lead to an increase in the optimal carbon tax. However, if $V_{s s s}>0$, then it is possible that increase in risk will lead to a decrease in the optimal carbon tax.

Let us compute $V_{s s s}\left(s^{*}, 0\right)$, using formula (10). This gives $V_{s s s}\left(s^{*}, 0\right)\left(r+\delta^{\prime}\left(s^{*}\right)\right)$ as

$$
\left\{u^{\prime \prime \prime}\left(c^{*}\right)\left[\frac{-V_{s s}}{u^{\prime \prime}\left(c^{*}\right)}\right]^{3}-D^{\prime \prime \prime}\left(s^{*}\right)+\delta^{\prime \prime \prime}\left(s^{*}\right) u^{\prime}\left(c^{*}\right)\right\}-3 \delta^{\prime \prime}\left(s^{*}\right) V_{s s} .
$$

Thus the sign of $V_{s s s}\left(s^{*}, 0\right)$ is ambiguous. It is negative if $u^{\prime \prime \prime}\left(c^{*}\right) \geqslant 0, D^{\prime \prime \prime}\left(c^{*}\right) \geqslant 0$ and $\delta^{\prime \prime \prime}\left(s^{*}\right) \leqslant 0$, since we know that $-3 \delta^{\prime \prime}\left(s^{*}\right) V_{s s} \leqslant 0$. But $V_{s s s}\left(s^{*}, 0\right)$ can be positive if $u^{\prime \prime \prime}\left(c^{*}\right) \leqslant 0, D^{\prime \prime \prime}\left(s^{*}\right) \leqslant 0$ and $\delta^{\prime \prime \prime}\left(s^{*}\right) \geqslant 0$ where at least one of these inequalities are strict, so that the term inside the curly brackets $\{\ldots\}$ is positive, and if this positive term dominates the term $\delta^{\prime \prime}\left(s^{*}\right) V_{s s}$. Thus, making use of (15) and (16) we can state the following proposition.

Proposition 2: (Effect of an increase in risk on the optimal carbon tax) Starting from a point in some neighbourhood of the risk-free steady state, an increase in risk will

(i) increase the carbon tax if $\sigma^{\prime}\left(s^{*}\right)>0, \delta^{\prime \prime \prime}\left(s^{*}\right) \leqslant 0, D^{\prime \prime \prime}\left(s^{*}\right) \geqslant 0$ and $u^{\prime \prime \prime}\left(c^{*}\right) \geqslant 0$;

(ii) decrease the carbon tax if $\delta^{\prime \prime \prime}\left(s^{*}\right) \geqslant 0, D^{\prime \prime \prime}\left(s^{*}\right) \leqslant 0$ and $u^{\prime \prime \prime}\left(c^{*}\right) \leqslant 0$ where at least one of these inequalities is strict, and both $3 \delta^{\prime \prime}\left(s^{*}\right) V_{\text {ss }}$ and $\sigma^{\prime}\left(s^{*}\right)$ are sufficiently small.

Proposition 2 highlights the important role of the third derivative of three functions, namely the utility function, $u(c)$, the damage function $D(s)$, and the decay function $\delta(s)$, in determining the effect of an increase in risk on optimal policy. This is in line with the literature on precautionary saving, where the third derivative of the utility function plays a fundamental role. In fact, Leland ${ }^{20}$ and Drèze and Modigliani $^{21}$ showed that uncertainty concerning future incomes leads the riskaverse consumer to increase savings if $u^{\prime \prime \prime}(c)>0$. An intuitive explanation is as

\footnotetext{
${ }^{20}$ Leland (1968).

${ }^{21}$ Drèze and Modigliani (1972).
} 
follows: $u^{\prime \prime \prime}>0$ means that the marginal utility schedule, $u^{\prime}(c)$, is a strictly convex and decreasing curve. Suppose the income in the future period $t$ is either $c_{L}-b$ or $c_{H}+b$ with probability $1 / 2$ each, where $b$ is a parameter. Regardless of the value of $b$, we have $E c_{t}=\left(c_{L}+c_{H}\right) / 2$. The strict convexity of $u^{\prime}$ implies

$$
E u^{\prime}\left(c_{t}\right)>u^{\prime}\left(E c_{t}\right) \text {. }
$$

The inequality (17) is known as Jensen's inequality. An increase in $b$ represents an increase in risk (i.e. an increase in the variance of $\left.c_{t}\right)$. It will increase $E u^{\prime}\left(c_{t}\right)$ because the schedule $u^{\prime}\left(\right.$.) is strictly convex. ${ }^{22}$ This increase in future expected marginal utility encourages savings.

This effect was further explored by Kimball, ${ }^{7}$ who used the term "prudence" to describe the attitude of individuals whose utility function exhibits the property that $u^{\prime \prime \prime}(c)>0$. Kimball ${ }^{7}$ showed that the precautionary effect is equivalent to a sure reduction in future income, which can be approximated by the product of two terms: (a) 50 per cent of the variance of the risk, and (b) an index of convexity of marginal utility. If the utility function has a constant relative risk aversion $\chi$, this index is equal to $1+\chi .^{23}$

Gollier $^{24}$ and Gollier and Weitzman ${ }^{25}$ found the importance of "prudence" in the so-called "extended Ramsey rule", which incorporates uncertainty into the relationship between the "correct consumption discount rate" and the familiar trio, namely the pure rate of preference for the present, the growth rate of expected consumption, and the elasticity of intertemporal substitution, $1 / \chi$. The general conclusion is that taking account of the precautionary effect, the consumption discount rate should be reduced when there is uncertainty in future growth rates. ${ }^{26}$

${ }^{22}$ If the utility function were quadratic, so that $u^{\prime \prime \prime}=0$, the marginal utility schedule $u^{\prime}(c)$ would be a downward sloping straight line, and the inequality sign in (17) would be replaced by an equality sign. Increase in uncertainty would then have no effect in savings. This explains the so-called certainty equivalence result in quadratic utility models of savings.

${ }^{23}$ Note that, in the standard framework, if $\chi$ is the relative risk aversion coefficient, then $1 / \chi$ is the elasticity of intertemporal substitution, though in a more general framework, this link is no longer a necessity (Epstein and Zin, 1991). Gollier (2011) assumed that coefficient of relative risk aversion $\chi$ is 2, in line with Arrow et al. (2004), Weitzman (2007b), and Nordhaus (2008). This implies that the elasticity of intertemporal substitution (EIS) is $1 / 2$, consistent with the range of estimates by macroeconomists. However, recently Barro (2009) argued that these estimates were biased, and that a good micro-based estimate for the EIS should be around 2. See also Gruber (2006).

${ }^{24}$ Gollier $(2002,2011)$.

${ }^{25}$ Gollier and Weitzman (2010).

26 The empirical magnitude of this reduction is a matter of contention. Gollier (2002) found that if one uses U.S. data on the volatility of the growth rate, the discount rate should be reduced by only one tenth of 1 per cent. In a more recent paper, Gollier (2011) argued that, for climate change problems, one should use data for developing economies, which are likely to be most adversely affected by climate change. He found that the appropriate consumption discount rate should be around 2.5 per cent instead of 4 per cent, assuming no serial correlation in the annual growth rate of consumption. (For the case with serial correlation, see Gollier (2008), and Weitzman (2007a). 
In our model, the effect of an increase in risk on optimal policy depends not only on $u^{\prime \prime \prime}$, but also on $D^{\prime \prime \prime}$ and $\delta^{\prime \prime \prime}$. This is not surprising, because our model is more complex than the classical Lucas-tree model used in, for example, Gollier. ${ }^{27}$ Furthermore, instead of risks about the exogenous growth rate of consumption in the Lucas-tree model, we deal with risks affecting one component of the growth rate of the pollution stock, $s$. This is where $D^{\prime \prime \prime}(s)$ and $\delta^{\prime \prime \prime}(s)$ become relevant. In Proposition 2(i), the condition on the sign of $\delta^{\prime \prime \prime}(s)$ is opposite to the condition on the sign of $u^{\prime \prime \prime}(c)$ and $D^{\prime \prime \prime}(s)$, because $\delta(s)$ represents the decay rate, not a utility function.

It is important to note that in this paper we do not address the issue of an appropriate consumption discount rate for evaluation of investment projects in pollution abatement, because the simple Lucas-tree framework does not apply here. This complex issue is a topic for further research.

Corollary 1: (Effect of an increase in risk on the optimal emission rate) Starting from a risk-free steady state, an increase in risk will

(i) decrease the optimal emission rate near the risk-free steady state if $\sigma^{\prime}\left(s^{*}\right)>0$, $\delta^{\prime \prime \prime}\left(s^{*}\right) \leqslant 0, D^{\prime \prime \prime}\left(s^{*}\right) \geqslant 0$ and $u^{\prime \prime \prime}\left(c^{*}\right) \geqslant 0$;

(ii) increase the optimal emission rate near the risk-free steady state if $\delta^{\prime \prime \prime}\left(s^{*}\right) \geqslant 0$, $D^{\prime \prime \prime}\left(s^{*}\right) \leqslant 0$ and $u^{\prime \prime \prime}\left(c^{*}\right) \leqslant 0$ where at least one of these inequalities is strict, and both $3 \delta^{\prime \prime}\left(s^{*}\right) V_{s s}$ and $\sigma^{\prime}\left(s^{*}\right)$ are sufficiently small.

Finally, we want to find out if an increase in risk will decrease the expected stationary stock of pollution. Strictly speaking, in the presence of the disturbance term $\sqrt{\mu} \sigma(s(t)) \mathrm{d} z$, there is no such thing as the stationary level of the stock. Nevertheless, we can ask the following question: at what stock level is the expected rate of change in the stock equal to zero? That is, we want to find a value $s^{* *}(\mu)$ such that $^{28}$

$$
c\left(s^{* *}, \mu\right)-\delta\left(s^{* *}\right)=0
$$

With a slight abuse of language, we will refer to the stock $s^{* *}(\mu)$ as the "expected stationary state". Note that by definition, $s^{* *}(0)=s^{*}$, the steady state stock of the risk-free scenario. Let us find out whether an increase in risk will result in a lower expected stationary state, that is, whether $\left(\mathrm{d} s^{* *} / \mathrm{d} \mu\right)<0$.

We can state the following result:

Proposition 3: (Effect of an increase in risk on the expected stationary pollution stock level) Assume that $\delta^{\prime}\left(s^{*}\right)>0$ and $D^{\prime \prime \prime}\left(s^{*}\right) \geqslant 0$. Then, starting from a point

${ }^{27}$ Gollier (2011).

${ }^{28}$ We use the notation $s^{* *}(\mu)$ to refer to the expected stationary state when $\mu>0$, to be distinguished from $s^{*}$, which is the steady-state stock under the risk-free scenario. 
in some small neighbourhood a risk-free steady state, an increase in risk will result in

(a) a fall in the expected stationary pollution stock level, if the conditions stated in case (i) of Proposition 2 hold.

(b) an increase in expected stationary pollution stock level, if the conditions stated in case (ii) of Proposition 2 hold.

\section{Non-cooperative game among $\boldsymbol{n}$ countries: Effect of a marginal increase in risk}

In the previous section, there is only one intertemporal maximiser. In other words, we have confined attention to a stochastic optimal control problem, and studied the effects of an increase in risk in that simple context. There are however many realworld situations where several intertemporal optimisers interact with each other in a strategic way: they engage in a dynamic non-cooperative game, in which the evolution of the state variable is influenced by the actions chosen by these players. The theory that provides solution concepts and toolboxes for analysing this type of problems is called the theory of differential game. ${ }^{11}$ This section investigates the extent to which the comparative-dynamics methodology of the previous section can be applied to differential games involving one state variable and several control variables (one for each player).

For concreteness, we use a specific setting, with a simple economic interpretation: a global pollution game, where emissions by $n$ countries contribute to the accumulation of the global pollution stock. Suppose each country attempts to maximise its intertemporal social welfare by solving an optimal control problem, knowing that other countries also seek to solve their optimal control problem in a similar way. Assume they do not cooperate with each other. How does an increase in risk affect their welfare?

We denote country $i$ 's emissions at time $t$ by $c_{i}(t)$. The pollution stock $s$ obeys the transition equation

$$
\mathrm{d} s=\left[c_{1}+c_{2}+\cdots+c_{n}-\delta(s)\right] \mathrm{d} t+\sqrt{\mu} \sigma(s) \mathrm{d} z .
$$

The function $\delta(s)$ is the net natural decay rate.

Suppose country $i$ guesses that all other countries $j \neq i$ use the same emission strategy:

$$
c_{j}=c^{g}(s, \mu),
$$

where the superscript $g$ in $c^{g}$ indicates that this is a guessed function. Then the HJB equation for country $i$ is

$$
r V^{i}(s, \mu)=\max _{c_{i}} K^{i}\left(s, c_{i}+(n-1) c^{g}(s, \mu), \mu\right),
$$


where $K^{i}\left(s, c_{i}, \mu\right)$ is the pseudo-Hamiltonian function:

$$
K^{i}\left(s, c_{i}, \mu\right) \equiv U\left(s, c_{i}\right)+V_{s}^{i}(s, \mu)\left[c_{i}+(n-1) c^{g}(s, \mu)-\delta(s)\right]+\frac{\mu \sigma^{2}(s)}{2} V_{s s}^{i} .
$$

We assume that $U$ is separable:

$$
U\left(s, c_{i}\right) \equiv u\left(c_{i}\right)-D(s) .
$$

Since this is a game, we must choose an equilibrium concept. Following Dockner et $a l^{11}$ and others, we will focus on the "Markov-perfect Nash equilibrium". In this equilibrium, each player $i$ (country $i$ ) chooses a strategy $c_{i}=c^{i}(s, \mu)$ that determines $c_{i}(t)$ as a function of the currently observed level of the stock, $s(t)$, such that its strategy is the best reply to the strategies $c^{j}(s, \mu)$ chosen by the other players $j \neq i$. Since the countries are identical, it is natural to focus on a symmetric Markov-perfect equilibrium, where all players use the same strategy.

\section{Symmetric Markov-perfect equilibrium}

Consider the optimal control problem of country $i$. Since $c_{i}(t)$ must maximise the pseudo-Hamiltonian function, the first-order condition is

$$
u^{\prime}\left(c_{i}\right)+V_{s}^{i}=0 .
$$

Given the value function $V^{i}(s, \mu)$, the first-order condition (20) yields the policy function $c_{i}=c^{i}(s, \mu)$. Thus, in a symmetric equilibrium, condition (20) yields the symmetric equilibrium strategy $c(s, \mu)$ :

$$
u^{\prime}(c(s, \mu))+V_{s}^{i}(s, \mu)=0 \Rightarrow c(s, \mu)=u^{\prime-1}\left[-V_{s}^{i}(s, \mu)\right] .
$$

Differentiate Eq. (21) with respect to $s$ to obtain the expression for the derivative $\partial c / \partial s$ of the equilibrium strategy $c(s, \mu)$ :

$$
u^{\prime \prime}(c(s, \mu)) \frac{\partial c(s, \mu)}{\partial s}+V_{s s}^{i}=0 .
$$

Thus

$$
\frac{\partial c(s, \mu)}{\partial s}=-\frac{V_{s s}^{i}}{u^{\prime \prime}(c(s, \mu))} .
$$

To obtain the derivative of the policy function with respect to $\mu$, differentiate the identity (21) with respect to $\mu$

$$
\begin{gathered}
u^{\prime \prime}(c(s, \mu)) \frac{\partial c(s, \mu)}{\partial \mu}+V_{s \mu}^{i}=0 \\
\operatorname{sign}\left[\frac{\partial c(s, \mu)}{\partial \mu}\right]=\operatorname{sign} V_{s \mu}^{i} .
\end{gathered}
$$


It turns out that, since we are dealing with a non-cooperative game, the determination of $V_{s s}^{i}, V_{s s s}^{i}$ and $V_{s \mu}^{i}$ is more complicated than in the case of a single decision-maker. The reason is that the envelope theorem is less helpful in the case of a non-cooperative game. To see the nature of the complication, note that the HJB equation (19) for each country involves the strategies used by other countries:

$$
r V^{i}(s, \mu)=u\left(c_{i}(s, \mu)\right)-D(s)+V_{s}^{i}\left[c_{i}(s, \mu)+(n-1) c^{g}(s, \mu)-\delta(s)\right]+\frac{\mu \sigma^{2}(s)}{2} V_{s s}^{i} .
$$

Differentiate Eq. (25) with respect to $s$, using the fact that all countries use the same strategy in equilibrium,

$$
\begin{aligned}
r V_{s}^{i}= & -D^{\prime}(s)-V_{s}^{i} \delta^{\prime}(s)+\left[\left(u^{\prime}+V_{s}^{i}\right) \frac{\partial c}{\partial s}\right]+(n-1) V_{s}^{i} \frac{\partial c}{\partial s}+V_{s s}^{i}\{n c(s, \mu)-\delta(s)\} \\
& +\mu \frac{\partial}{\partial s}\left\{V_{s s}^{i}(s, \mu) \frac{\sigma^{2}(s)}{2}\right\} .
\end{aligned}
$$

Evaluating at $\mu=0$ and $s=s^{*}$, after substituting for (22), we obtain

$$
r V_{s}^{i}\left(s^{*}, 0\right)=-D^{\prime}-V_{s}^{i} \delta^{\prime}-(n-1) V_{s}^{i} \frac{V_{s s}^{i}}{u^{\prime \prime}(c(s, \mu))} .
$$

Thus

$$
D^{\prime}\left(s^{*}\right)=-V_{s}^{i}\left\{r+\delta^{\prime}\left(s^{*}\right)+\frac{(n-1) V_{s s}^{i}}{u^{\prime \prime}(c(s, \mu))}\right\}
$$

Then, at the risk-free steady state $s^{*}$, the following equality holds

$$
\frac{D^{\prime}\left(s^{*}\right)}{r+\delta^{\prime}\left(s^{*}\right)+\frac{(n-1) V_{S s}^{i}\left(s^{*}, 0\right)}{u^{\prime \prime}\left(\frac{\delta\left(s^{*}\right)}{n}\right)}}=u^{\prime}\left(\frac{\delta\left(s^{*}\right)}{n}\right) \text {. }
$$

This equation determines $s^{*}$, if the function $V^{i}(s, 0)$ is known. ${ }^{29}$

The interpretation of Eq. (27) is as follows: each country $i$ equates, at the steady state, the marginal utility of consumption (emissions) to the discounted value of the stream of future damages that it will suffer, where the discounting takes into account not only the discount rate $r$ but also the facts that (i) the added emissions will decay away, and (ii), as $i$ emits more, thus increasing the stock of pollution, other countries will respond by emitting less.

\footnotetext{
${ }^{29}$ Note however that, in differential games, there is a possibility that there are many (locally defined) Markov-perfect Nash equilibriums (MPNEs), and each of them will give rise to a different value function. See, for example, Dockner and Long (1993), and Rowat (2007). In what follows, we insist on globally defined MPNE, and we assume that it is unique.
} 
Let us compute the derivative $V_{s s}^{i}(s, \mu)$ by differentiating $V_{s}^{i}(s, \mu)$.

$$
r V_{s s}^{i}=-D^{\prime \prime}-V_{s}^{i} \delta^{\prime \prime}-V_{s s}^{i} \delta^{\prime}+V_{s s}^{i}\left[n \frac{\partial c}{\partial s}-\delta^{\prime}\right]+V_{s s s}^{i} G+(n-1)\left[V_{s s}^{i} \frac{\partial c}{\partial s}+V_{s}^{i} \frac{\partial^{2} c}{\partial s^{2}}\right] .
$$

Evaluating at the steady state

$$
r V_{s s}^{i}\left(s^{*}, 0\right)=-D^{\prime \prime}-V_{s}^{i} \delta^{\prime \prime}+V_{s s}^{i}\left[n \frac{\partial c}{\partial s}-2 \delta^{\prime}\right]+(n-1)\left[V_{s s}^{i} \frac{\partial c}{\partial s}+V_{s}^{i} \frac{\partial^{2} c}{\partial s^{2}}\right],
$$

where $\partial c / \partial s$ can be replaced by $-V_{s s} / u^{\prime \prime}$. The complication is that the above equation involves the term $\partial^{2} c / \partial s^{2}$. Without further information, to evaluate this term is not a trivial exercise. One consolation is that if the underlying utility function, damage function and decay function are quadratic, then we can expect that $c(s, \mu)$ is linear, in which case the term $\partial^{2} c / \partial s^{2}$ is simply zero. We will return to this special case later.

Can increases in risk improve welfare in a non-cooperative dynamic game?

Under non-cooperative abatement policies, does an increase in risk reduce welfare? or can it increase welfare? The answer depends on the strategic effect. To see this, differentiate Eq. (25) with respect to $\mu$

$$
r V_{\mu}^{i}=\left[u^{\prime}(c)+V_{s}\right] \frac{\partial c}{\partial \mu}+(n-1) V_{s}^{i} \frac{\partial c}{\partial \mu}+V_{s s}^{i}(s, \mu) \frac{\sigma^{2}(s)}{2}+V_{s \mu}^{i} G+\mu V_{s s \mu}^{i}(s, \mu) \frac{\sigma^{2}(s)}{2}
$$

Evaluated at $\mu=0$, the above equation becomes

$$
r V_{\mu}^{i}=(n-1) V_{s}^{i} \frac{\partial c}{\partial \mu}+V_{s s}^{i}(s, \mu) \frac{\sigma^{2}(s)}{2}+V_{s \mu}^{i} G .
$$

At the risk-free steady state, $G=0$, hence evaluated at the non-cooperative steady state $s^{*}$

$$
r V_{\mu}^{i}\left(s^{*}, 0\right)=(n-1) V_{s}^{i} \frac{\partial c}{\partial \mu}+V_{s s}^{i}\left(s^{*}, 0\right) \frac{\sigma^{2}(s)}{2} .
$$

This result is in sharp contrast to Result 1 (which applies to the case of a central planner).

Proposition 4: When n countries play a non-cooperative game, $V_{\mu}^{i}$ and $V_{s s}^{i}$ do not necessarily have the same sign (in sharp contrast to the social planner case). Therefore, in the context of a non-cooperative game, an increase in risk may raise the welfare of every country.

This is an interesting result. Unlike the social planner case, in the case of a noncooperative game, even if $V_{s s}^{i}<0$, an increase in risk may increase welfare if the 
positive strategic term $(n-1) V_{s}^{i}(\partial c / \partial \mu)$ is sufficiently large to outweigh the negative term $V_{s s}<0$.

The intuition behind this result is as follows. An increase in risk has two effects on a country's welfare. The direct effect of risk on welfare is a harmful one, given that countries are risk averse. The indirect effect of an increase in risk is beneficial, since it induces countries to reduce their emissions, thus mitigating the tragedy of the commons. Now, since $V_{s}^{i}$ is negative in the case of a public bad, the term $(n-1) V_{s}^{i}(\partial c / \partial \mu)$ is positive if and only if $(\partial c / \partial \mu)$ is negative, that is, if and only if an increase in risk induces each country to emit less.

\section{Effect of marginal increase in risk in a linear-quadratic model}

To show that Proposition 4 is not empty, in this subsection we make some additional assumptions so that we can directly compute the value function $V^{i}(s, \mu)$ and obtain the sign of $\partial c / \partial \mu$. We assume that the functions $\delta(s)$ and $\sigma(s)$ in Eq. (18) take the forms

$$
\delta(s)=\theta s, \quad \sigma(s)=g \sqrt{s},
$$

where $\theta>0$ and $g>0$. Furthermore, let the utility function and the damage function be quadratic

$$
\begin{gathered}
u(c)=A c-\frac{1}{2} c^{2}, A>0, \\
D(s)=\kappa s+\frac{h}{2} s^{2}, \kappa>0, h>0 .
\end{gathered}
$$

We assume that $\kappa$ is small, such that

$$
A(\theta+r)-\kappa>0
$$

It is convenient to define

$$
\pi \equiv 2(A(\theta+r)-\kappa)>0
$$

Given these specific functional forms, the symmetric equilibrium strategy for this stochastic dynamic game must be linear in the state variable ${ }^{30}$ :

$$
c(s, \mu)=\eta-\alpha s .
$$

We can show that, in the symmetric equilibrium,

$$
\eta=\frac{\pi+\left((n-1)-\mu g^{2}\right) \alpha}{2(\theta+r+(2 n-1) \alpha)},
$$

\footnotetext{
${ }^{30}$ This is because we insist that the strategy must be defined for all non-negative values of $s$.
} 
where $\eta>0$ if $\mu g^{2}$ is small, and

$$
\alpha=\frac{-(2 \theta+r)+\sqrt{(2 \theta+r)^{2}+4 h(2 n-1)}}{2(2 n-1)}>0 .
$$

We make the following assumption:

Assumption B1: Assume that $\mu g^{2}$ is small enough such that the following inequality holds

$$
\mu g^{2}<(n-1)+\left(\frac{\pi}{\alpha}\right)
$$

This assumption ensures that Nash equilibrium emission is strictly positive when the stock of pollution is near zero.

Note that $\alpha$ is independent of $\mu$, and $\eta$ is a decreasing function of $\mu$. Under this equilibrium strategy, the value function of each country is

$$
V^{i}(s, \mu)=-\frac{\alpha}{2} s^{2}-[A-\eta(\mu)] s+\phi(\mu),
$$

where

$$
\phi(\mu) \equiv \frac{1}{2 r}\left\{A^{2}+(2 n-1)[A-\eta(\mu)]^{2}-2 A n[A-\eta(\mu)]\right\}
$$

The evolution of pollution stock follows the Cox-Ingersoll-Ross process ${ }^{31}$ such that, starting at time zero with the initial stock $s_{0}$, the expected value of $s(t)$ is

$$
E(s(t))=s_{0} e^{-(\theta+n \alpha) t}+\frac{n \eta(\mu)}{\theta+n \alpha}\left[1-e^{-(\theta+n \alpha) t}\right] .
$$

Eq. (36) implies that the expected pollution stock converges to a steady state defined by

$$
s^{* *}(\mu)=\frac{n \eta(\mu)}{\theta+n \alpha}>0 .
$$

We can state the following propositions:

Proposition 5: (Effect of an increase in risk on equilibrium strategies)

(i) There exists a symmetric Markov-perfect equilibrium, in which all countries use the same linear emission strategy

$$
c_{i}=\eta(\mu)-\alpha s,
$$

where $\eta(\mu)$ and $\alpha$ are given by eqs. (31) and (32).

(ii) An increase in risk makes all countries reduce their emissions, at any level of the pollution stock.

\footnotetext{
${ }^{31}$ Athanassoglou (2010).
} 
Remark on emission tax rates: From Eq. (34) we find that the marginal value of the stock of pollution is negative:

$$
V_{s}^{i}=-\alpha s-[A-\eta(\mu)]<0 .
$$

If in each economy all private agents do not take into account the effects of their individual emissions on the country's aggregate emission, the government of each country $i$ must impose an emission tax at the rate $\tau^{i}(t)$ per unit at time $t$, in order to achieve its Nash equilibrium emission, where

$$
\tau(t)=-V_{s}^{i}(s(t), \mu)>0
$$

The effect of an increase in risk on the emission tax can then be calculated as follows:

$$
\frac{\mathrm{d} \tau(t)}{\mathrm{d} \mu}=-\eta^{\prime}(\mu)=\frac{\alpha g^{2}}{2(\theta+r+(2 n-1) \alpha)}>0
$$

We state this result as a corollary:

Corollary 2 Countries respond to an increase in risk by increasing the tax rate on emissions.

Finally, we can state the following result concerning welfare:

Proposition 6: (Effect of an increase in risk on Nash equilibrium welfare)

(i) If $n=1$, then an increase in risk reduces welfare.

(ii) If $n \geqslant 2$, then starting at $s_{0}=0$, an increase in risk increases welfare if and only if the following condition holds:

$$
\mu g^{2} \alpha+2 \kappa>\frac{2 A n(\theta+r)}{2 n-1},
$$

(iii) If $n \geqslant 2$, and starting at $s_{0}>0$, an increase in risk increases welfare if and only if the following condition holds:

$$
\mu g^{2} \alpha+2 \kappa>\frac{2 A n(\theta+r)}{2 n-1}+2 r s_{0}(\theta+r+(2 n-1) \alpha) .
$$

\section{Conclusion}

We have developed a model of global pollution and used a simple toolbox based on the dynamic envelope theorem ${ }^{32}$ to investigate the effects of a marginal increase in risk on welfare and on optimal policies. As expected, the third derivatives of utility

\footnotetext{
${ }^{32}$ Kimball (2014).
} 
function, damage function and decay function play an important role in determining the sign of the policy responses. When we extended the model to the case where there are several agents acting strategically in a dynamic game, we found that the application of the dynamic envelope theorem is of limited usefulness. In this case, we had to make additional assumptions about functional forms to facilitate the evaluation of the effects of a marginal increase in risk. We found that, even if agents are risk averse, an increase in risk may increase their welfare. This is because the greater risk induces agents to be more conservative, and this results in reduced exploitation of the global common.

\section{Acknowledgements}

The authors are grateful to Mike Hoy, Miles Kimball, Ray Rees and Achim Wambach for helpful comments.

\section{References}

Athanassoglou, S. (2010) 'Dynamic nonpoint source pollution control policy: Ambient transfer and uncertainty', Journal of Economic Dynamics and Control 34(12): 2494-2509.

Arrow, K.J., Dasgupta, P., Goulder, L., Daily, G., Ehrlich, P., Heal, G., Levin, S., Mäler, K.-G., Schneider, S., Starrett, D. and Walker, B. (2004) 'Are we consuming too much?', Journal of Economic Perspectives 18(3): 147-172.

Barro, R. (2009) 'Rare disasters, asset prices, and welfare costs', American Economic Review 99(1): 243-264.

van den Bremer, T., van der Ploeg, R. and Wills, S. (2013) The elephant in the ground: Managing oil and sovereign wealth, typescript, Department of Economics, University of Oxford.

Caputo, M.R. (1990) 'Comparative dynamics via envelope methods in variational calculus', Review of Economic Studies 57(4): 689-697.

Caputo, M.R. (2003) 'The comparative dynamics of closed-loop controls for discounted infinite horizon optimal control problems', Journal of Economic Dynamics and Control 27(8): 1335-1365.

Caputo, M.R. (2005) Foundations of Dynamic Economic Analysis, New York: Cambridge University Press.

Caputo, M.R. (2007) 'The envelope theorem for locally differentiable Nash equilibria of finite horizon differential games', Games and Economic Behavior 61(2): 198-224.

Caputo, M.R. and Ling, C. (2013) 'The intrinsic comparative dynamics of locally differentiable feedback Nash equilibria in autonomous and exponentially discounted infinite horizon differential games', Journal of Economic Dynamics and Control 37(10): 1982-1994.

Dockner, E., Jorgensen, S., Long, N.V. and Sorger, G. (2000) Differential Games in Economics and Management Science, Cambridge: Cambridge University Press.

Dockner, E. and Long, N.V. (1993) 'International pollution control: Cooperative versus noncooperative strategies', Journal of Environmental Economics and Management 25(1): 13-29.

Drèze, J.H. and Modigliani, F. (1972) 'Consumption decisions under uncertainty', Journal of Economic Theory 5(3): 308-335.

Epstein, L.G. and Zin, S. (1991) 'Substitution, risk aversion and the temporal behavior of consumption and asset returns: An empirical framework', Journal of Political Economy 99(2): 263-286.

Gollier, C. (2002) 'Time horizon and the discount rate', Journal of Economic Theory 107(2): 463-473. 
Gollier, C. (2008) 'Discounting with fat-tailed economic growth', Journal of Risk and Uncertainty 37(2): 171-186.

Gollier, C. (2011) 'On the underestimation of precautionary effect in discounting', The Geneva Risk and Insurance Review 36(2): 95-111.

Gollier, C. and Weitzman, M.L. (2010) 'How should the distant future be discounted when discount rate are uncertain?', Economics Letters 107(3): 350-353.

Gruber, J. (2006) A tax-based estimate of the elasticity of intertemporal substitution, NBER working paper 11945.

Kimball, M.S. (1990) 'Precautionary savings in the small and in the large', Econometrica 58(1): 53-73.

Kimball, M.S. (2014) 'The effects of uncertainty on optimal control models in the neighborhood of a steady state', The Geneva Risk and Insurance Review 39(1): 2-39 (Paper presented at the EGRIE Conference, Paris, September 2013.).

LaFrance, J.T. and Barney, L.D. (1991) 'The envelope theorem in dynamic optimization', Journal of Economic Dynamics and Control 15(2): 355-385.

Leland, H.E. (1968) 'Savings the uncertainty: The precautionary demand for savings', Quarterly Journal of Economics 82(3): 456-473.

Leonard, D. and Long, N.V. (1992) Optimal Control Theory and Static Optimization in Economics, Cambridge: Cambridge University Press.

Ling, C. and Caputo, M.R. (2011) 'A qualitative characterization of symmetric open-loop Nash equilibria in discounted infinite horizon differential games', Journal of Optimization Theory and Applications 149(1): 151-174.

Long, N.V. (1979) 'Two theorems on generalized diminishing returns and their applications to economic analysis', Economic Record 55(148): 58-63.

Merton, R.C. (1990) Continuous Time Finance, Oxford: Basil Blackwell.

Nordhaus, W.D. (2008) A Question of Balance: Weighing the Options on Global Warming Policies, New Haven, CT: Yale University Press.

Rowat, C. (2007) 'Non-linear strategies in linear-quadratic differential games', Journal of Economic Dynamics and Control 31(10): 3179-3202.

Sandal, L.K., Steinshamn, S.I. and Grafton, R.Q. (2003) 'More is less: The tax effects of ignoring flow externalities', Resource and Energy Economics 25(3): 239-254.

Turnovsky, S.J. (2000) Methods of Economic Dynamics, 2nd edn, Cambridge, MA: MIT Press.

Weitzman, M.L. (2007a) 'Subjective expectations and asset-return puzzle', American Economic Review 97(4): 1102-1130.

Weitzman, M.L. (2007b) 'A review of the Stern review on the economics of climate change', Journal of Economic Literature 45(3): 703-724.

\section{Appendix}

\section{Proof of Proposition 3}

Recall that

$$
u^{\prime}(c(s, \mu))=-V_{s}(s, \mu)
$$

and

$$
r V(s, \mu)=u(c(s, \mu))-D(s)+V_{s}(s, \mu)[c(s, \mu)-\delta(s)]+\frac{\mu \sigma^{2}(s)}{2} V_{s s}(s, \mu)
$$


Then

$$
V_{s}=-D^{\prime}(s)+V_{s s}[c(s, \mu)-\delta(s)]-V_{s} \delta^{\prime}(s)+\mu \frac{\mathrm{d}}{\mathrm{d} s}\left[\frac{\sigma^{2}(s)}{2} V_{s s}(s, \mu)\right] .
$$

Setting $c\left(s^{* *}, \mu\right)-\delta\left(s^{* *}\right)=0$, we obtain

$$
V_{s}\left(s^{* *}, \mu\right)\left(r+\delta^{\prime}\left(s^{* *}\right)\right)=-D^{\prime}\left(s^{* *}\right)+\mu \frac{\mathrm{d}}{\mathrm{d} s}\left[\frac{\sigma^{2}\left(s^{* *}\right)}{2} V_{s s}\left(s^{* *}, \mu\right)\right] .
$$

Thus

$u^{\prime}\left(\delta\left(s^{* *}\right)\right)\left(r+\delta^{\prime}\left(s^{* *}\right)\right)=D^{\prime}\left(s^{* *}\right)-\mu\left[\sigma\left(s^{* *}\right) \sigma^{\prime}\left(s^{* *}\right) V_{s s}\left(s^{* *}, \mu\right)+\frac{\sigma^{2}\left(s^{* *}\right)}{2} V_{s s s}\left(s^{* *}, \mu\right)\right]$.

This equation yields $s^{* *}=s^{* *}(\mu)$. (In the limiting case where $\mu=0$, we have $s^{* *}(0)=s^{*}$.)

The effect of an increase in $\mu$ on $s^{* *}$ is given by

$$
\begin{aligned}
& \left\{\frac{\partial}{\partial s}\left[u^{\prime}\left(\delta\left(s^{* *}\right)\right)\left(r+\delta^{\prime}\left(s^{* *}\right)\right)-D^{\prime}\left(s^{* *}\right)\right]\right\} \mathrm{d} s^{* *} \\
& +\mu\left\{\frac{\partial}{\partial s}\left[\sigma\left(s^{* *}\right) \sigma^{\prime}\left(s^{* *}\right) V_{s s}\left(s^{* *}, \mu\right)+\frac{\sigma^{2}\left(s^{* *}\right)}{2} V_{s s s}\left(s^{* *}, \mu\right)\right]\right\} \mathrm{d} s^{* *} \\
& =-\left[\sigma\left(s^{* *}\right) \sigma^{\prime}\left(s^{* *}\right) V_{s s}\left(s^{* *}, \mu\right)+\frac{\sigma^{2}\left(s^{* *}\right)}{2} V_{s s s}\left(s^{* *}, \mu\right)\right] \mathrm{d} \mu \\
& -\mu\left\{\frac{\partial}{\partial \mu}\left[\sigma\left(s^{* *}\right) \sigma^{\prime}\left(s^{* *}\right) V_{s s}\left(s^{* *}, \mu\right)+\frac{\sigma^{2}\left(s^{* *}\right)}{2} V_{s s s}\left(s^{* *}, \mu\right)\right]\right\} \mathrm{d} \mu
\end{aligned}
$$

Evaluating this expression at $\mu=0$, so that $s^{* *(0)}=s^{*}$, we obtain

$$
\frac{\mathrm{d} s^{* *}}{\mathrm{~d} \mu}=\frac{-\left[\sigma\left(s^{*}\right) \sigma^{\prime}\left(s^{*}\right) V_{s s}\left(s^{*}, 0\right)+\frac{\sigma^{2}\left(s^{*}\right)}{2} V_{s s s}\left(s^{*}, 0\right)\right]}{\frac{\partial}{\partial s}\left[u^{\prime}\left(\delta\left(s^{*}\right)\right)\left(r+\delta^{\prime}\left(s^{*}\right)\right)-D^{\prime}\left(s^{*}\right)\right]}
$$

The numerator has the sign of $-V_{\mu s}\left(s^{*}, 0\right)$. The sign of the denominator is

$$
u^{\prime \prime}\left(c^{*}\right) \delta^{\prime}\left(s^{*}\right)\left(r+\delta^{\prime}\left(s^{*}\right)\right)+u^{\prime}\left(c^{*}\right) \delta^{\prime \prime}\left(s^{*}\right)-D^{\prime \prime \prime}\left(s^{*}\right)
$$

which is negative if $\delta^{\prime}\left(s^{*}\right)>0$ and $D^{\prime \prime \prime}\left(s^{*}\right) \geqslant 0$. 


\section{About the Authors}

Quentin Grafton is Professor of Economics and Director of the Centre for Water Economics, Environment and Policy at the Crawford School of Public Policy of the Australian National University.

Tom Kompas is the Director of the Crawford School of Public Policy at the Australian National University and Director of the Australian Centre for Biosecurity and Environmental Economics.

Ngo Van Long is James McGill Professor of Economics at McGill University. 